\title{
Rhodium-Catalyzed cis-Selective Hydrogenation of Fluoroarenes
}

Metal-Catalyzed

Asymmetric

Synthesis and

Stereoselective

Reactions

\section{Key words}

rhodium catalysis

hydrogenation

stereoselectivity

fluoroarenes

fluorocycloalkanes

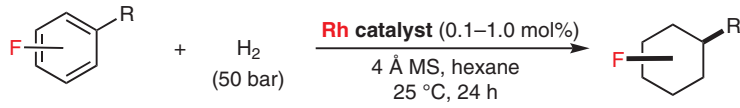<smiles>FC1CCCCC1F</smiles><smiles>CC1CCCC(F)C1F</smiles><smiles>FC1CC(F)CC(F)C1</smiles>

$90 \%$ yield $\quad 96 \%$ yield $\quad 81 \%$ yield $\quad 65 \%$ yield<smiles>FC1C(F)C(F)C(F)C(F)C1F</smiles>
$d r=17: 1 \quad d r=6: 1 \quad d r>20: 1$<smiles>[18OH]C1CCC(F)CC1</smiles><smiles>O=S(=O)(O)NC1CCC(F)CC1</smiles><smiles>CC(=O)C1CCC(F)CC1</smiles><smiles>O=C1NCCC2CCC(F)CC12</smiles>

$34 \%$ yield 93\% yield $\mathrm{dr}=13: 1$ $88 \%$ yield $81 \%$ yield
dr $=6: 1$<smiles>FC1CC(F)C(F)CC1F</smiles><smiles>FC1CCC(F)C(F)C1F</smiles><smiles>FC1CC(F)C(F)C(F)C1F</smiles>

pplication of the fluorinated cycloalkane:

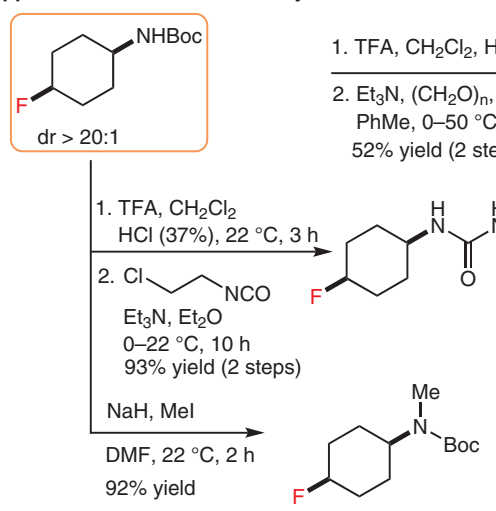
$\mathrm{HBF}_{4}(40 \%)$, glyoxal ${ }^{\circ} \mathrm{C}, 12 \mathrm{~h}$

\section{$\begin{array}{ll}80 \% \text { yield } & 83 \% \text { yield } \\ d r=9: 1 & \text { d }\end{array}$}

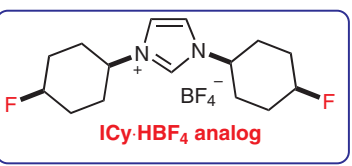

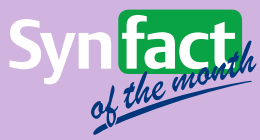

Significance: All-cis-polyfluorinated cycloalkanes exhibit attractive electronic properties due to their high dipole moments. However, multistep syntheses from diastereoselectively fluorinated precursors are generally required. The authors report a rhodium/cyclic (alkyl)(amino)(carbene) complex catalyzed cis-selective hydrogenation of fluorinated arenes to provide a variety of highly diastereoselectively fluorinated cycloalkanes.
Comment: To suppress the formation of hydrodefluorinated byproducts, the choice of a less-polar solvent such as hexane is important.

SYNFACTS Contributors: Hisashi Yamamoto, Takahiro Sawano 